\title{
Detection of Macrosegregation in a Large Metallic Specimen Using XRF
}

\author{
E.J. Pickering ${ }^{a}$, M. Holland ${ }^{b}$
}

a Corresponding Author.

Department of Materials Science and Metallurgy, University of Cambridge, Pembroke Street, Cambridge CB2 3QZ, UK

ejp57@cam.ac.uk

${ }^{b}$ Geotek Ltd, 4 Sopwith Way, Daventry, NN11 8PB, UK

melanie@geotek.co.uk 


\section{Abstract}

The characterisation of macroscopic chemical segregation in engineering components over length scales of many metres can be an arduous task. This report investigates the implentation of a technique that is capable of mapping long-range variations in the chemical composition of metal components, without the need for extensive sample preparation. The capability of the method is optimised for large production components, in a setting where process parameters such as measurement time and minimal surface preparation are of importance. Hence, a readily-available hand-held X-ray fluorescence instrument and analysis software are used to map macrosegregation in a low-alloy steel slab.

\section{Introduction}

Macrosegregation in metals has been of concern to the manufacturers of large cast products for many decades, and its prediction and mitigation have proven to be formidable challenges. ${ }^{1)}$ This segregation is that which occurs over distances similar to the dimensions of the casting, and arises during solidification due to the movement of microsegregated solid and liquid. ${ }^{1-3)}$ In the case of large ingots and sand castings, the distances involved may be of the order of centimetres or metres.

The segregation is important because it leads to deterioration and scatter in mechanical properties, and the location of solute-rich regions can be detrimental to subsequent fabrication processes such as welding. ${ }^{4)}$ The detection of segregation over large distances is therefore a useful capability, permitting the control of quality in manufactured parts, and providing much-needed information and guidance to those modelling macrosegregation phenomena. Techniques such as energy-dispersive or wavelength-dispersive X-ray analysis in the electron microscope, optical-emission spectroscopy, combustion analysis and wet chemical methods are common chemical analysis tools, but are often destructive, time-consuming and are only able to analyse small volumes, thus rendering them impractical for large components. To map segregation in the past, these analysis methods have been used on small samples extracted from the ingot at regularly-spaced intervals (i.e., via trepanning), with the data extrapolated to produce composition maps. ${ }^{5-8)}$ Such a method may not detect small regions of heavily-segregated material, and is both expensive and requires the removal of significant quantitites of material. X-ray fluorescence (XRF) spectroscopy, on the other hand, can be used to build up a detailed picture of composition over large distances, without the component being enclosed in a vacuum or destroyed beyond basic surface preparation.

XRF spectroscopy has its origins in the pioneering investigations into the use of X-rays carried out a century ago, but it is only over more recent decades that significant advances in instrumentation and analysis have been made which have led to its wider application. ${ }^{9,10)}$ Now XRF is used as an analysis technique in applications as diverse as medical and biological imaging, ${ }^{11)}$ art and archaelogical examination, ${ }^{12)}$ scrap metal sorting, and the analysis of geological cores and soil samples. ${ }^{13)}$ Specialist instruments and data analysis methods have allowed for the detection of minute concentrations of all but a few of the lightest elements (down to the microscopic scale), whilst the mass-produced handheld analysers commonplace in industry are used for less specialised purposes. It is true to say that automated XRF mapping is not a new technique (it is used, for example, in geological core logging and artwork examination, ${ }^{12,13)}$ ) and has recently been applied to the mapping of solute distribution in metals at a large-scale fixed facility by Volz et al. $\left.{ }^{14}\right)$ However, the particular system used here differs in several key ways to that used by Volz et al.: it employs standard off-the-shelf components, can be expanded to larger sizes (limited by robotics only), it can make measurements on surfaces which are not ideally flat (i.e., the detector can move vertically), and the equipment can be moved relatively straightforwardly. 
In this work, we examine the use of a readily-available XRF instrument to detect and map macrosegregation in large metal sections. Emphasis is placed upon investigating its pragmatic application, rather than the on the precise calibration of the equipment for accurate quantitative analysis. Such calibrations require significant experimental undertakings and expertise (for example, see Lachance and Classie ${ }^{15)}$ and Rousseau ${ }^{16)}$ ), and instead it is assumed these calibrations have been sufficiently accomplished by the supplier of the XRF equipment (it would generally be very difficult for a casting engineer to fully optimise their XRF technique for every alloy they encounter). The effect of dwell times and surface finish on segregation detection, and the reproducibility of the data, are examined. The primary aim was to qualitatively identify the minimum dwell times and surface preparations required to reliably detect segregates.

\section{Experimental}

An Olympus Innov-X Delta Dynamic hand-held XRF analyser was employed, with robotics designed and constructed by Geotek Ltd. ${ }^{17}$ ) The analyser and robotics were controlled using the following software packages: Geotek's Multi-Sensor Core Logging XYZ, ${ }^{18)}$ Microsoft ActiveSync ${ }^{19)}$ and Innov$\mathrm{X}$ Delta Advanced PC. ${ }^{20)}$ The analyser incorporated a Rh source and a $10 \mathrm{~mm}$ silicon drift detector. Fig. 1 shows the experimental set-up. The analyser was standardised using a reference metallic sample supplied by Olympus. A low-alloy steel slab, known to contain small areas of enriched material, was used as the test piece for all investigations. This sample was taken from a forged production component, which had been cast as part of a 200-tonne ingot. The enriched regions were characterised as being channel segregates, also known as A-segregates, that penetrated the thickness of the slab. ${ }^{1,21)}$ Fig. 3 shows this slab after grinding and etching in 5\% nitric acid; note that the A-segregates appear elongated in one direction because of the forging process. Table 1 gives the composition of the bulk material and enriched regions as measured using wavelength-dispersive X-ray spectroscopy, implemented in a Cameca SX-100 electron probe micro analyser. During investigations, the surface of this slab was prepared to four different finishes, shown in Table 2, with the roughness measured using a Veeco Dektak 6M Stylus Profiler. Energy-dispersive X-ray analysis (EDX) was carried out using a field emission gun scanning electron microscope, CamScan MX2600.

The hand-held XRF was used in a mode that utilises two X-ray beams, the first of energy $40 \mathrm{kV}$ and current $52 \mu \mathrm{A}$ to detect transition metals, and the second with energy $13 \mathrm{kV}$ and current 20 $\mu \mathrm{A}$ for lighter elements such as $\mathrm{Mg}, \mathrm{Al}, \mathrm{Si}, \mathrm{P}$ and S. Olympus' own peak-fitting and quantisation algorithms were employed via the Innov-X software. The raw spectra were also retained.

\section{Results}

\subsection{Spatial Resolution}

The spatial resolution of the XRF instrument was investigated using an Fe-Ni dissimilar metal bond produced using a diffusion-bonding process at $1050^{\circ}$ for 30 minutes. ${ }^{22)}$ EDX revealed that the interdiffusion layer produced was approximately $20 \mu \mathrm{m}$ in width. Fig. 2 shows the blurring of the composition profile due to spatial resolution of the XRF instrument, and was obtained by gradually stepping across the interface using a $1 \mathrm{~mm}$ step size. It is evident that the vast majority of characteristic X-rays are collected from a region 5-7 $\mathrm{mm}$ in width in both $x$ and $y$. 


\subsection{Mapping}

A preliminary map of the slab's surface chemistry was obtained using both analytical X-ray beams. The live time for the $40 \mathrm{kV}$ beam was set to $30 \mathrm{~s}$ ( $42 \mathrm{~s}$ real time), whilst the live time for the $13 \mathrm{kV}$ beam was $2.5 \mathrm{~s}$ ( $60 \mathrm{~s}$ real time). The surface of the slab was prepared to Roughness $\mathrm{C}$ in Table 2, and the sample measured in the unetched state. A step size of $5 \mathrm{~mm}$ was used throughout the work. Maps for $\mathrm{Cr}, \mathrm{Mn}, \mathrm{Ni}, \mathrm{Mo}$ and $\mathrm{Si}$ are shown in Fig. 3. It is clear that segregation features are most readily distinguished for Mo and $\mathrm{Mn}$, and are also discernible in the maps for $\mathrm{Ni}$ and $\mathrm{Cr}$, despite poorer contrast in these cases. For Si there is no clear identification of segregated material, and the map appears much noisier. Comparing absolute concentrations to those in Table 1, it is evident that the values for $\mathrm{Cr}, \mathrm{Mn}, \mathrm{Ni}$ and Mo are close to the EPMA values, whilst the Si concentration measured is significantly lower. Maximum values should not be expected to tally with the values for $c_{*}$ in Table 1 due to the area over which measurements are taken, i.e., segregated features do not completely fill the sampling window and hence are less prominent than would be the case with a better spatial resolution.

\subsection{Dwell-Time Variation}

The effect of dwell time variation was investigated to determine whether using a small dwell time would consistently lead to the detection of segregated material. The small region of the slab highlighted in Fig. 3 was measured with a retained surface finish given by Roughness $\mathrm{C}$ in Table 2. Only the $40 \mathrm{kV}$ analytical beam was utilised, in light of the apparent difficulty in measuring Si, meaning that only $\mathrm{Cr}, \mathrm{Mn}, \mathrm{Ni}$ and Mo were measured. An indication of the reproducibility of segregate detection was found by triplicating measurements on this region, i.e., making three measurement runs over the same region, as shown in Fig. 4. Detected variations of Mo are by far the most reproducible, with the location of enriched material readily apparent at dwell times as low as $1 \mathrm{~s}$. Mn results are informative and reasonably consistent with $10 \mathrm{~s}$ dwell, whilst $\mathrm{Ni}$ appears to require $30 \mathrm{~s}$ and $\mathrm{Cr}$ closer to $60 \mathrm{~s}$.

\subsection{Surface Roughness}

To examine the effect of surface roughness on the reproducibility of measurements, the surface finishes listed in Table 2 were used and the region highlighted in Fig. 3 analysed once again. Only the $40 \mathrm{kV}$ beam was used with a $30 \mathrm{~s}$ dwell time, and runs were triplicated. Fig. 5 shows the results. It is evident that, within the range of roughness assessed, the surface finish had little significant effect on the reproducibility of results. Adequate detection is achieved even with the roughest surface finish. Note that because material was removed between measurements, it cannot be guaranteed the segregated material analysed was always in precisely the same position or the same shape (see results for Roughness C vs Roughness D), but no significant changes in composition were detected when layers were removed - this is a natural result of channel-type segregation - and hence the reproducibility can still be compared.

\section{Discussion}

Concentrations in XRF analysis are calculated using the net peak signal, i.e., the difference in the measured peak signal and the measured background signal. The standard counting error associated with the net signal is therefore influenced not only by the error in the peak signal, $\sigma_{p}$, but also by the error in the background signal, $\sigma_{b}$. The total error in the net signal, $\sigma_{n e t}$ (i.e., the noise), can 
be found by the following expression, which is a result of assuming the distributions of peak and background values are Gaussian and independent:

$$
\sigma_{n e t}^{2}=\sigma_{p}^{2}+\sigma_{b}^{2}
$$

It is known that X-ray emission is a probabilistic process which can be described by Poisson statistics. A standard result of this is that, at sufficiently high count rates, the Poisson distribution of the total number of counts approaches a Gaussian distribution. The theoretical standard deviation in this distribution, $\sigma_{*}$, is a function of the total number of counts, $C$, as given by $\sigma_{*}=\sqrt{C}$. Therefore, the combined standard deviation in the net signal can be expressed:

$$
\sigma_{n e t}=\sqrt{C_{p}+C_{b}}
$$

where $C_{p}$ and $C_{b}$ are the total peak and background counts. It is this noise in the signal that prevents the detection of small changes in it, and hence influences the detection of changes in concentration of an element. The change in signal due to a real change in concentration must be discernable over the noise in order to be reproducibly detected. This simple framework can be used to help explain, qualitatively here, the effect of dwell times and surface roughness on segregation detection. It is true to say that a statistical analysis could have been used to calculate the required dwell time to detect a certain variation (i.e., the number of counts needed to ensure the noise is small enough to reliably detect a particular change in signal) rather than embarking on the replication of measurements. However, the calculation through which the Innov-X software finds concentrations will be far more complex, taking into account factors including atomic number effects, cross-fluoresence, absorption and background profile. The errors associated with these will all contribute to the final uncertainty, potentially changing it significantly, and hence no such statistical calculations were made. Instead, an empirical study of reproducibility was undertaken with the aim of identifying whether sensible dwell times could be used.

It was found that for the steel studied, variations in composition of Mo could be most readily and reproducibly revealed over the range of dwell times and surface finishes studied. This capability then decreased from $\mathrm{Mn}$, to $\mathrm{Ni}$, to $\mathrm{Cr}$, and finally Si. Table 3 gives an indication of the relative magnitudes of the maximum and background number of counts for the elements analysed, with Fig. 6 showing these graphically (the true signal is, of course, all the counts which are taken to produce the peak, not just the maximum value). It is clear that by considering counting statistics alone, the error for $\mathrm{Si}$ should be greatest due to the high level of the background (a result of Compton X-rays at low energies) with a modest peak signal. Mo and Mn have high peak-to-background ratios, and high peak signals, delivering improved signal-to-noise ratio. The peak signals for $\mathrm{Cr}$ and $\mathrm{Ni}$ are lower, delivering relatively more noise. The $\mathrm{Ni}$ signal is low due to absorption of characteristic X-rays by the iron matrix. ${ }^{23)}$

The better detection of segregated material found at longer dwell times was simply a result of the improved signal-to-noise ratio delivered by a higher number of counts. The effect is most pronounced for elements with a low baseline number of counts, i.e., $\mathrm{Cr}$ and $\mathrm{Ni}$. The apparent insensitivity of results to surface roughness variations can also be explained in a similar manner: changing the roughness did not significantly affect the number of registered counts or the background signal, for any element analysed (the total number of counts dropped by only $\sim 12 \%$ between the smoothest and roughest surfaces). The use of specific abrasives during sample surface preparation can lead to significant contamination issues in metal XRF, particularly in the case of $\mathrm{Si}^{23)}$ but are unlikely to have been significant here ( $\mathrm{SiC}$ grinding paper was used for Roughness $\mathrm{A}$, but was followed by diamond paste stages, and the grinding and milling procedures used no Si-based abrasives). In summary, variations in concentration measured by XRF are not only influenced by real changes in sample chemistry (Table 1), but are also affected by a number of other factors that cannot be ignored when interpreting results. 


\section{$5 \quad$ Practical Implications}

It is clear, and not surprising, that more consistent and accurate results can be obtained by increasing the number of characteristic X-rays counted by the detector. However, the aim of this investigation was to determine whether reproducible detection of segregates could be achieved using practical dwell times and surface finishes. Although the material examined in this study has been a highly-specific example, it has been demonstrated that the segregation present can be readily detected using dwell times of only a few seconds, particularly if species like Mo are targeted. This is certainly of great importance for potential incorporation into quality-control procedures. Longer dwells can lead to dramatic improvements in results - with $30 \mathrm{~s}$ dwell, variations of the order of a few hundred ppmw are reproducibly measured for Mo - and it is therefore capable of detecting long-range gradual variations if necessary. The same cannot be said for $\mathrm{Si}$, however, and it is clear that to significantly improve results would require prohibitively long dwell times if the same experimental apparatus were used. In practice, the required dwell time will be dependent on the magnitude of concentration variations and particular element of interest.

The insensitivity of results to surface finish is a promising result, as the careful preparation of large surfaces areas would be a costly task. It is likely surface finish will only become important for the accurate quantitiative detection of minute concentrations and light elements (the depth from which characteristic X-rays escape is much lower for lighter elements, and hence surface contamination and scattering become more important. ${ }^{23)}$ ) In terms of potential spatial resolution for the technique, the $5 \mathrm{~mm}$ sampling interval used here will have meant some material will have been sampled more than once, diluting results. It would theoretically be possible to improve the resolution of the technique to the magnitude of the step size if a 'reference' section of material had an known concentration profile and the spatial X-ray sampling profile of the detector was know, but this is not of concern here. Nevertheless, an XRF instrument capable of switching the size of its sampling window, from broad to very small, will shortly become available and may be of use to quickly detect segregated areas and then precisely analyse them. ${ }^{24)}$ Another distinct possibility is the incorporation of other analysis techniques, such as optical imaging and hardness measurements, into the same automated robotics, thereby yielding further useful information concurrently.

\section{Summary and Conclusions}

It was found that an automated XRF technique which employed readily-available equipment was capable of accurately and reproducibly detecting the macrosegregation present in a low-alloy steel sample. Adequate detection was achieved at dwell times of only a few seconds for some elements, and results appeared insensitive to the surface finishes investigated. There is no reason why the technique cannot also be used to assess long-range gradual variations, and there is potential for other measurement technqiues to be combined with XRF using the same automated mapping apparatus. Such a system could readily form part of a manufacturing quality-control procedure.

\section{Acknowledgements}

This work was undertaken as part of a project sponsored by Rolls-Royce Power Engineering plc (Submarines) in collaboration with Sheffield Forgemasters International. The author is greatly indebted to Dr Peter Schultheiss and others at Geotek Ltd for their support and supply of the automated XRF apparatus. The author also wishes to extend his thanks to Dr Amir Shirzadi for the preparation of diffusion-bonded samples, to James Nygaard for his assistance in carrying out surface roughness 
measurements, to Professor Harry Bhadeshia for helpful comments during the preparation of this manuscript, and to Professor Lindsay Greer for provision of laboratory facilities and electron microscopes.

\section{References}

[1] E.J. Pickering: 'Macrosegregation in Steel Ingots: The Applicability of Modelling and Characterisation Techniques', ISIJ, 2013, 53, 939-949.

[2] M.C. Flemings: 'Solidification Processing', 1974, McGraw-Hill.

[3] J.A. Dantzig and M. Rappaz: 'Solidification', 2009, EPFL Press.

[4] G. Ostberg: 'A Review of Present Knowledge Concerning the Influence of Segregates on the Integrity of Nuclear Pressure Vessels', International Journal of Pressure Vessels and Piping, 1997, 74, 153-158.

[5] C. Maidorn and B. Blind: 'Solidification and Segregation in Heavy Forging Ingots', Nuc. Eng. Des., 1985, 84, 285-296.

[6] H. Combeau, M. Zaloznik, S. Hans, and P.E. Richy: 'Prediction of Macrosegregation in Steel Ingots: Influence of the Motion and the Morphology of Equiaxed Grains', Metall. Mater. Trans. $B, 2009$, 40B, 289-304.

[7] R. Tanzer, W. Schutzenhofer, G. Reiter, H.P. Fauland, L. Konozsy, A. Ishmurzin, M. Wu, and A. Ludwig: 'Validation of a Multiphase Model for the Macrosegregation and Primary Structure of High-Grade Steel Ingots', Metall. Mater. Trans. B, 2009, 40B, 305-311.

[8] A.K. Singh, B. Basu, and A. Ghosh: 'Experimental Investigation of Macrosegregation During Vertical Solidification of Lead-Tin Alloys', Trans. Indian Inst. Metall., 2012, 65, 85-96.

[9] H.G.J. Moseley: 'The High-Frequency Spectra of the Elements', Phil. Mag., 1913, 26, 1024-34; 1914, 27, 703-713.

[10] R. Jenkins: 'X-Ray Fluorescence Spectroscopy', 2nd edition, 1999, John Wiley \& Sons Inc.

[11] T. Paunesku, S. Vogt, J. Maser, B. Lai, and G. Woloschak: 'X-Ray Fluorescence Microprobe Imaging in Biology and Medicine'. J. Cell. Biochem., 2006, 99, 1489-1502.

[12] M. Mantler and M. Schreiner: 'X-Ray Fluorescence Spectrometry in Art and Archaeology'. X-Ray Spectrom., 2000, 29, 3-17.

[13] R.G. Rothwell (ed): 'New Techniques in Sediment Core Analysis', Geological Society Special Publication No. 267, 2006, The Geological Society.

[14] H.M. Volz, G.J. Havrilla, R. M. Aikin Jr., and V.M. Montoya: 'Macroscopic X-ray Fluorescence Capability for Large-Scale Elemental Mapping', "Advances in X-Ray Analysis," Proc. 2010 Denver X-Ray Conf., Aug. 1-6, 2010, Denver, CO, USA, 2010.

[15] G.R. Lachance and F. Claisse: 'Quantitative X-Ray Fluorescence Analysis', 1995, John Wiley \& Sons, Ltd.

[16] R.M. Rousseau: 'Detection Limit and Estimate of Uncertainty of Analytical XRF Results', The Rigaku Journal, 2001, 18, 33-47. 


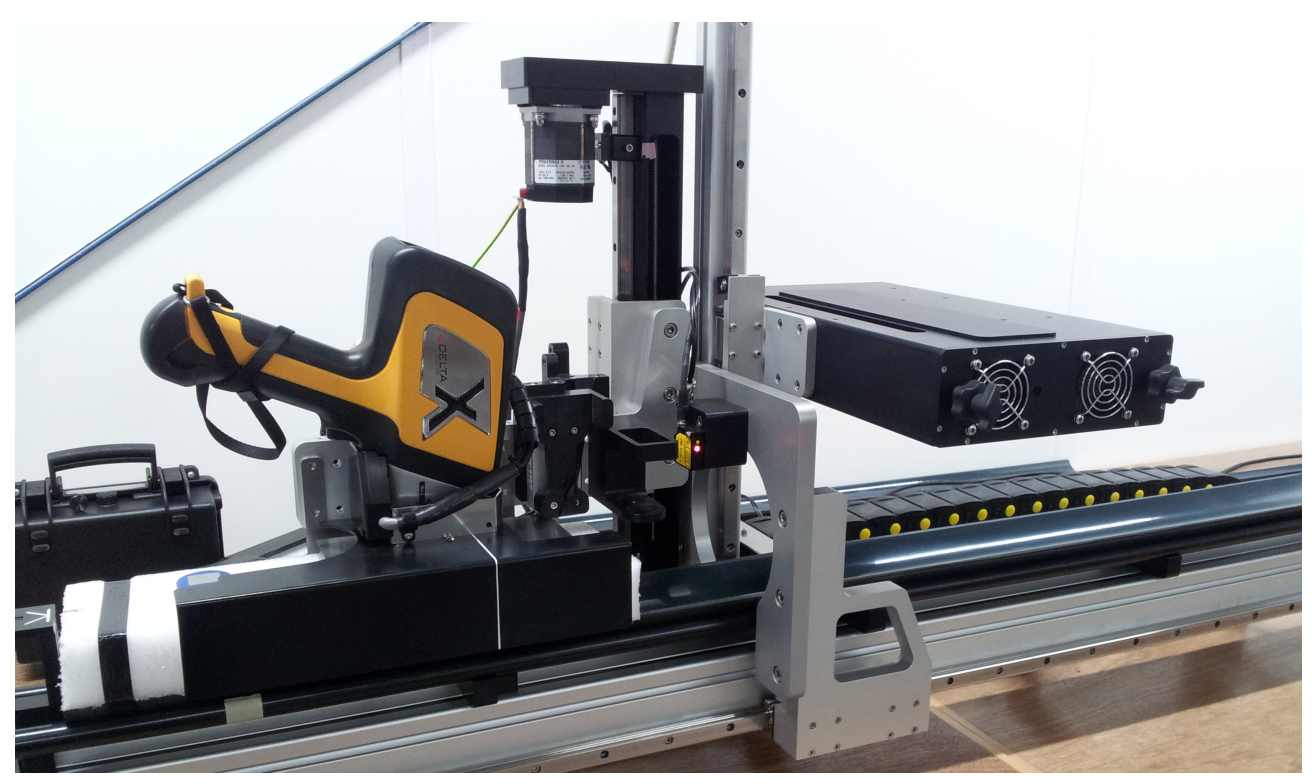

Figure 1: The automated XRF apparatus. A standard hand-held XRF is mounted on a robotic gantry system with laser-guided movement.

[17] Geotek Ltd, Daventry, UK, http://www.geotek.co.uk/.

[18] Geotek MSCL XYZ sofware, version 3.4. Geotek Ltd, Daventry, UK.

[19] Microsoft@ActiveSync@Version 4.5.0 (Build 5096), Microsoft Corporation.

[20] Innov-X Delta Advanced PC Software, Olympus Corporation.

[21] E.J. Pickering and H.K.D.H. Bhadeshia: 'The Consequences of Macroscopic Segregation on the Transformation Behaviour of a Pressure-Vessel Steel', Proceedings of the ASME 2013 Pressure Vessels 83 Piping Division Conference, July 14-18, 2013, Paris, France, 2013, in press.

[22] A.A. Shirzadi: 'Novel Method for Diffusion Bonding Superalloys and Aluminium Alloys', USA Patent 6,669,534 B2, December 2005; UK Patent 2380491, July 2005.

[23] V.E. Burke, R. Jenkins, and D.K. Smith: 'A Practical Guide for the Preparation of Specimens for X-Ray Fluoresence and Z-Ray Diffraction Analysis'. 1998, John Wiley \& Sons, Inc.

[24] P. Schultheiss, Geotek Ltd, private communication.

\section{$7 \quad$ Figures and Tables}




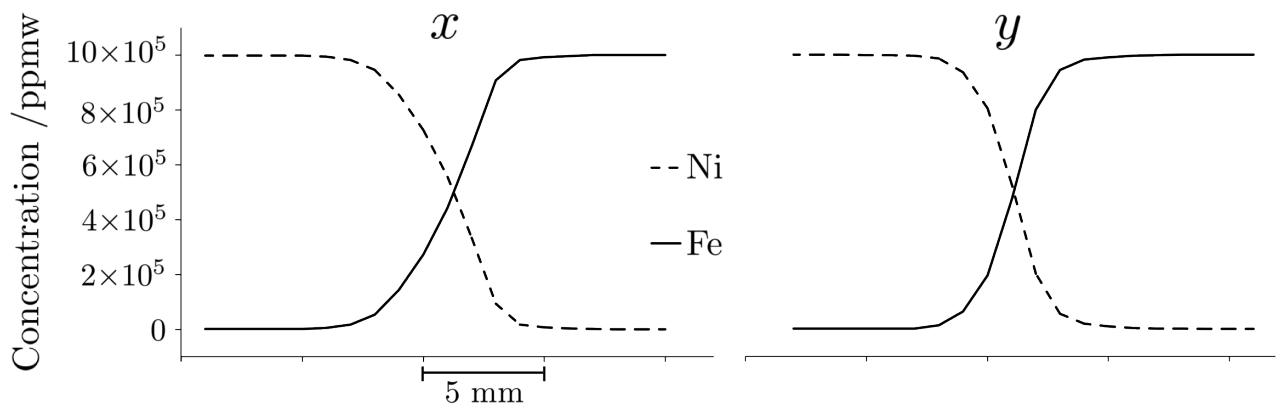

Figure 2: Measurement of the spatial resolution of the XRF instrument using a Fe-Ni dissimilar metal bond and a $1 \mathrm{~mm}$ step size.

Table 1: Chemical compositions of the bulk and enriched material, combining the results of several broad-beam $(40 \mu \mathrm{m})$ electron microprobe spot-analyses. The uncertainties in parentheses, represent one standard deviation.

\begin{tabular}{lccccc}
\hline & \multicolumn{5}{c}{ Concentration / ppmw } \\
& $\mathrm{Cr}$ & $\mathrm{Mn}$ & $\mathrm{Ni}$ & $\mathrm{Mo}$ & $\mathrm{Si}$ \\
\hline \multirow{2}{*}{ Bulk $\left(c_{0}\right)$} & $\mathbf{2 2 0 0}$ & $\mathbf{1 2 8 0 0}$ & $\mathbf{6 9 0 0}$ & $\mathbf{4 7 0 0}$ & $\mathbf{2 4 0 0}$ \\
& $(100)$ & $(400)$ & $(190)$ & $(100)$ & $(100)$ \\
& & & & & \\
Enriched $\left(c_{*}\right)$ & $\mathbf{2 6 0 0}$ & $\mathbf{1 5 9 0 0}$ & $\mathbf{8 6 0 0}$ & $\mathbf{6 2 0 0}$ & $\mathbf{2 8 0 0}$ \\
& $(100)$ & $(700)$ & $(400)$ & $(600)$ & $(100)$ \\
$c_{*} / c_{0}$ & 1.18 & 1.24 & 1.25 & 1.32 & 1.17 \\
\hline
\end{tabular}



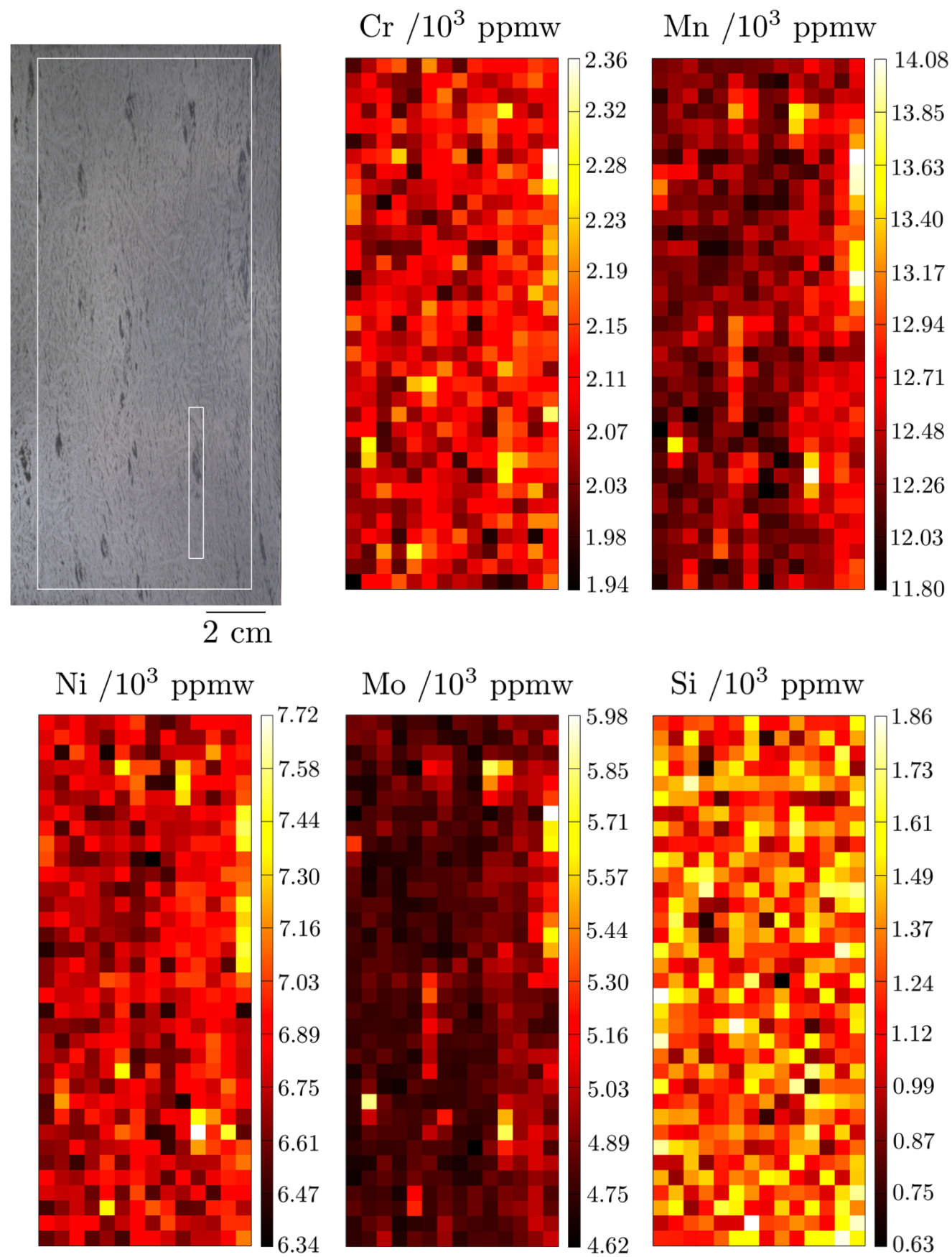

Figure 3: A photograph of the etched low-alloy steel slab with areas of analysis highlighted by white boxes. Alongside, the coloured images correspond to composition maps from the area inside the larger white box. The area inside the smaller white box was used for the maps presented in Fig. 4 . 

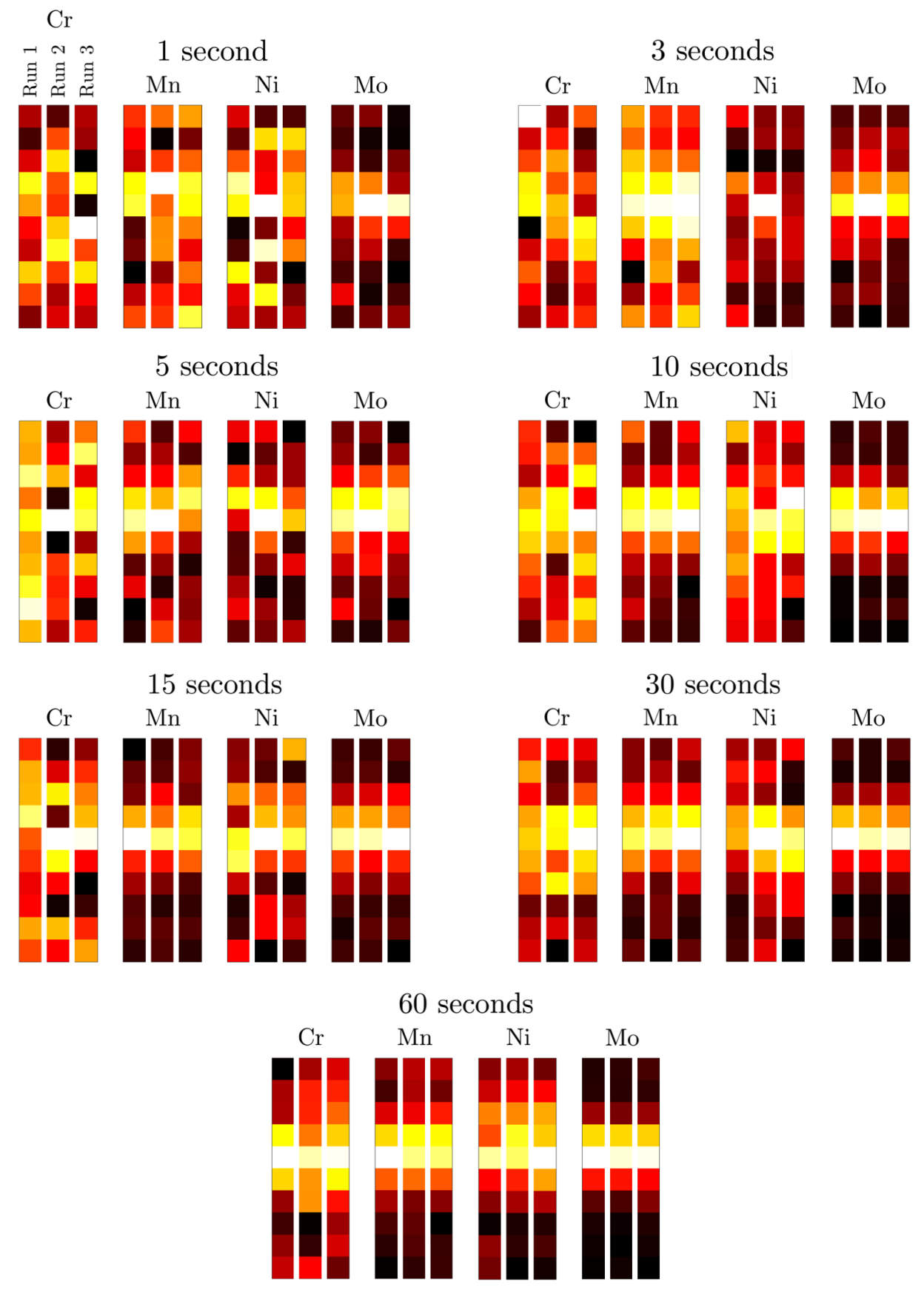

Figure 4: Results of dwell-time investigations. Measurements of $\mathrm{Cr}, \mathrm{Mn}, \mathrm{Ni}$ and Mo were triplicated for each dwell time on the small region highlighted in Figure 3. Note that a different concentration scale is used for each dwell time due to the changes in range - in general the lower dwell times produced a higher range of detected concentrations due to increased noise. 


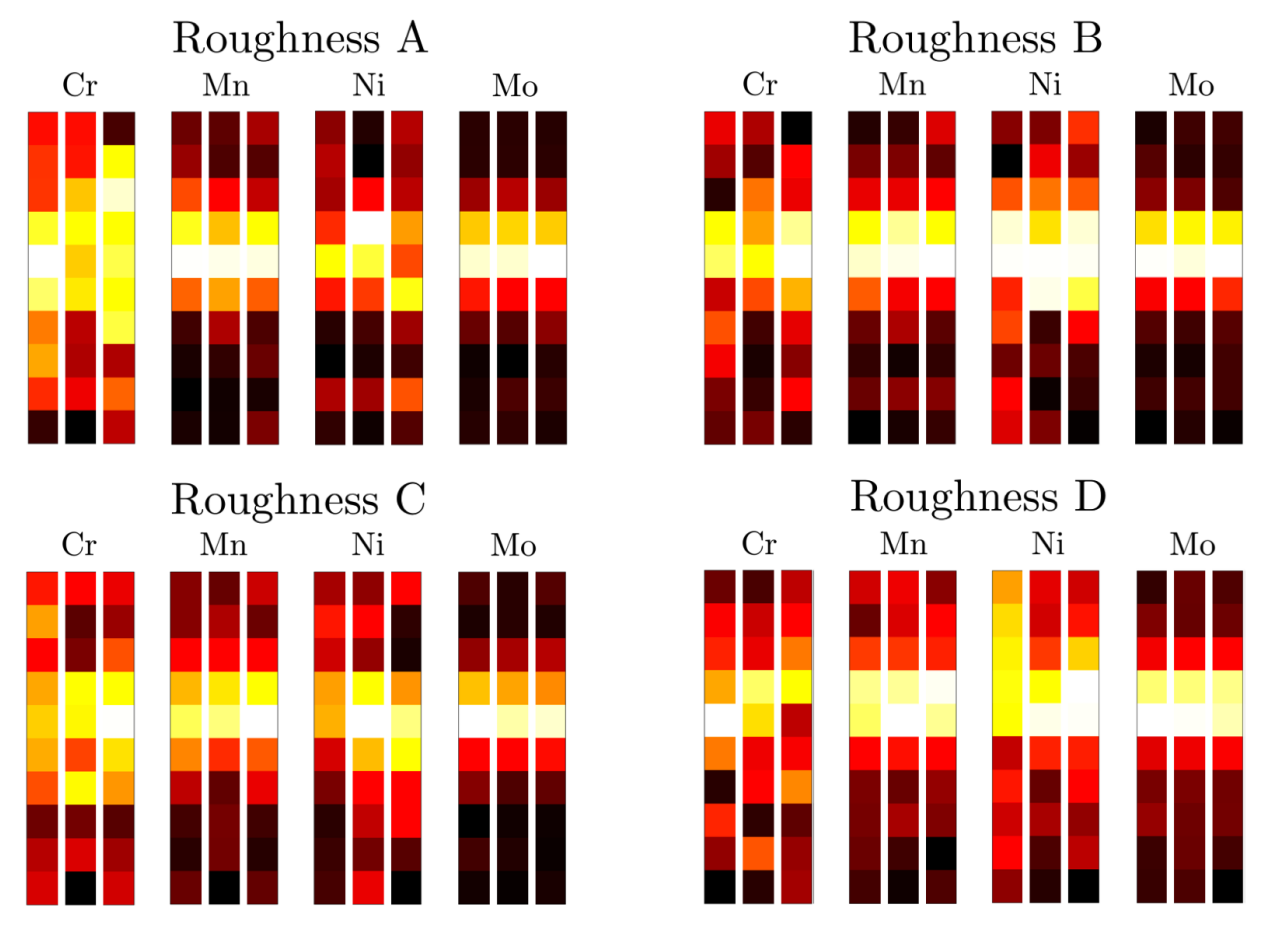

Figure 5: Results of surface-roughness investigations.

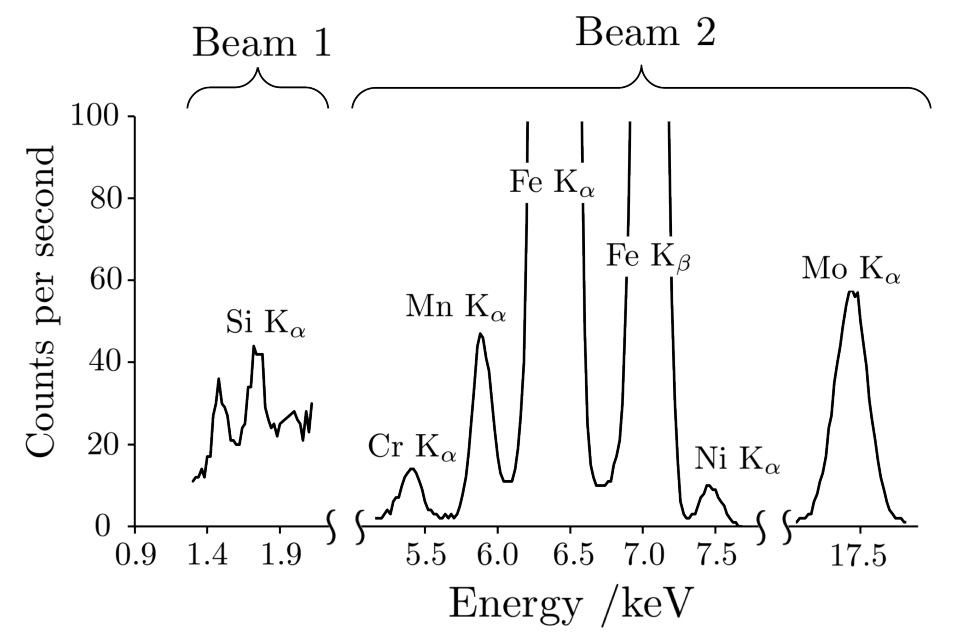

Figure 6: Characteristic peaks for the elements analysed. Note that although the absolute number of counts for the Si $\mathrm{K}_{\alpha}$ peak is relatively high, it is not compared to the peak height for $\mathrm{Fe} \mathrm{K}_{\alpha}$ using Beam 2: the maximum values for the $\mathrm{Fe}_{\alpha}$ peak for Beam 1 and Beam 2 were 3794 and 94292 counts per s respectively. The peak signal for each element will have been a function of both element concentration and the strength of X-ray interaction (i.e., characteristic X-ray yield). 
Table 2: Surface roughness data. For each surface finish, four $2.5 \mathrm{~mm}$ scans were made in two perpendicular directions, and the mean result found. Error given is one standard deviation of the mean result. The stylus tip radius used was $12.5 \mu \mathrm{m} . \mathrm{R}_{\mathrm{a}}$ is the mean deviation centreline, whilst $\mathrm{R}_{\mathrm{t}}$ is the maximum peak-valley distance.

\begin{tabular}{cccl}
\hline Label & $\mathrm{R}_{\mathrm{a}} / \mu \mathrm{m}$ & $\mathrm{R}_{\mathrm{t}} / \mu \mathrm{m}$ & Method of Surface Preparation \\
\hline $\mathrm{A}$ & $0.038 \pm 0.008$ & $0.429 \pm 0.099$ & SiC grinding, diamond polishing \\
$\mathrm{B}$ & $1.546 \pm 0.782$ & $7.286 \pm 3.256$ & Milling with WC tip (fine) \\
$\mathrm{C}$ & $2.118 \pm 0.818$ & $13.982 \pm 6.333$ & Surface grinding with $\mathrm{Al}_{2} \mathrm{O}_{3}$ disc \\
$\mathrm{D}$ & $6.723 \pm 3.265$ & $29.849 \pm 13.498$ & Milling with WC tip (coarse) \\
\hline
\end{tabular}

Table 3: X-ray count spectrum data for the elements analysed. The peak maximum is given as a relative guide for the strength of the signal, whilst the peak minimum is given as an approximate guide to the influence of the background and/or other characteristic peaks which may have made quantitative calculations more challenging.

\begin{tabular}{lcc}
\hline Peak and beam & $\begin{array}{c}\text { Peak maximum } \\
\text { /counts per s }\end{array}$ & $\begin{array}{c}\text { Peak minimum } \\
\text { /counts per s }\end{array}$ \\
\hline $\mathrm{Cr} \mathrm{K}_{\alpha}$, Beam 1 & 14 & 2 \\
$\mathrm{Mn} \mathrm{K}_{\alpha}$, Beam 1 & 47 & 2 \\
$\mathrm{Ni} \mathrm{K}_{\alpha}$, Beam 1 & 10 & 0 \\
$\mathrm{Mo} \mathrm{K}_{\alpha}$, Beam 1 & 58 & 1 \\
$\mathrm{Si} \mathrm{K}_{\alpha}$, Beam 2 & 44 & 20 \\
\hline
\end{tabular}

